\title{
Image-driven discriminative and generative methods for establishing microstructure- processing relationships relevant to nuclear fuel processing pipelines
}

Elizabeth Kautz ${ }^{1}$, Wufei Ma ${ }^{2}$, Arun Baskaran ${ }^{2}$, Aritra Chowdhury ${ }^{3}$, Vineet Joshi ${ }^{1}$, Bulent Yener $^{2}$ and Daniel Lewis ${ }^{2}$

${ }^{1}$ Pacific Northwest National Laboratory - PNNL, Richland, Washington, United States, ${ }^{2}$ Rensselaer Polytechnic Institute, Troy, New York, United States, ${ }^{3}$ GE Global Research, Niskayuna, New York, United States

Low enriched uranium alloyed with $10 \mathrm{wt}$ \% molybdenum (U-10Mo) has been identified as a promising alternative to highly enriched uranium oxide dispersion fuels for use in high performance research and test reactors. Manufacturing U-10Mo alloy fuel involves several complex thermomechanical processing steps, as schematically described in Figure 1, and understanding of the microstructure and its evolution throughout the various fabrication steps is critical to enable the deployment of a reliable fuel production capability.

This work explores the applicability of image-driven machine learning methods [1] to developing microstructure-processing relationships. Specifically, we seek to understand the role of several thermomechanical processing steps in the microstructure evolution observed in the U-10Mo system. An improved approach to determining microstructure-processing relationships is developed and presented, involving feature extraction, segmentation, and classification using a random forest model [2]. Microstructure image data are segmented to identify microstructural features of interest and quantify area fraction of these features, including the $\gamma$-UMo matrix, uranium carbide, and DP reaction transformation products.

Several experiments were considered to explore metrics of microstructure representations, specifically how well information such as area fractions of phases, their spatial arrangement, and micrograph texture impact classification accuracies. We refer to these metrics as area and spatial features. Area features are the area fraction of each phase/microstructural feature of interest. Spatial features are computed by first measuring the following for each region (matrix, carbide, lamellar transformation products): the $x$ and $y$ coordinates of the centroid, area (in square pixels), and the ratio of area of the region to the area of its bounding box. Spatial features are simply a concatenation the following measures: the number of regions in a bounding box, the mean and standard deviation of the areas, the standard deviation of the centroid coordinates, and the mean and standard deviation of area ratios.

Experimentation revealed that in a 10-class classification problem, characterization of micrographs using area features only resulted in a F1 score of $62.4 \%$. When spatial and area features are combined, the model performance is improve and a F1 score of $78.9 \%$ is achieved. Next, area and spatial features were combined with image texture (e.g., Haralick, local binary patterns, etc.) to describe image data, resulting in the highest F1 score of $95.1 \%$. This increased performance indicates that although the area features have long been regarded as a strong indication of the microstructure-processing relationships, we find that the predictive power of area features is actually very limited, and increased performance can be obtained by including spatial and texture information.

The application of generative adversarial networks (GANs) is also discussed as an emerging method for microstructure image generation. Our work has implications for quantitative microstructure analysis and 
development of microstructure-processing relationships in limited datasets typical in materials science studies, particularly in the nuclear materials domain.

Two different GAN architectures were tested (progressively growing GAN, or pg-GAN and Pix2Pix GAN). Example micrographs generated from pg-GAN are given in Figure 2. We find that although the synthetic images have reasonable qualitative agreement with real images, we cannot interpret the role of the input noise vectors in the generation of synthetic images. This lack of understanding prevents us from understanding how samples from different processing histories are distributed in the learned space of microstructure images or possibly revealing their underlying connections. The ability of GANs to generate realistic microstructures was tested by training a model to identify whether a microstructure is either synthetic or real. With fivefold cross-validation, an accuracy of $\sim 50 \%$ is achieved. Since the model fails to distinguish between features collected from real images and synthetic images, this finding suggests that the pg-GAN learned the underlying distribution of microstructure well.

In addition to the discriminative and generative methods developed, several challenges and best practices associated with recognition and quantification of microstructure image data in the interdisciplinary of machine learning for materials science will be discussed.

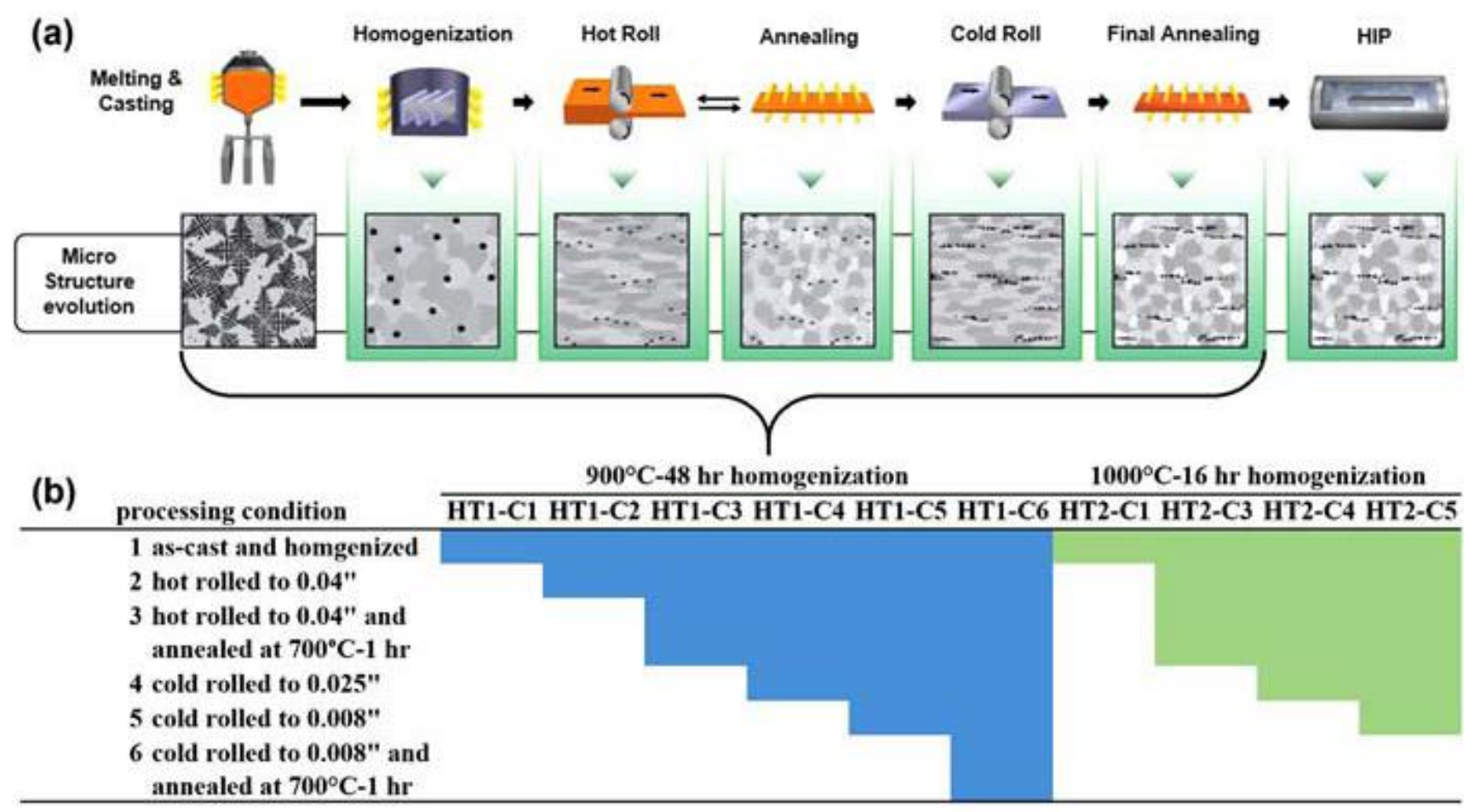

Figure 1. (a) Schematic of U-10Mo fuel fabrication, where the steps shown in the bracket were used to generate microstructure imaged and analyzed in this work. Sample conditions are indicated by homogenization treatment (HT) number, where HT-1 refers to $900^{\circ} \mathrm{C}-48 \mathrm{~h}$ and $\mathrm{HT}-2$ refers to $1000 \circ \mathrm{C}-16 \mathrm{~h}$. The processing condition (C) the micrographs represent is indicated by $\mathrm{C}$ followed by a number that corresponds to the number processing condition (e.g., $\mathrm{C} 1$ is a sample in the as-cast condition). 

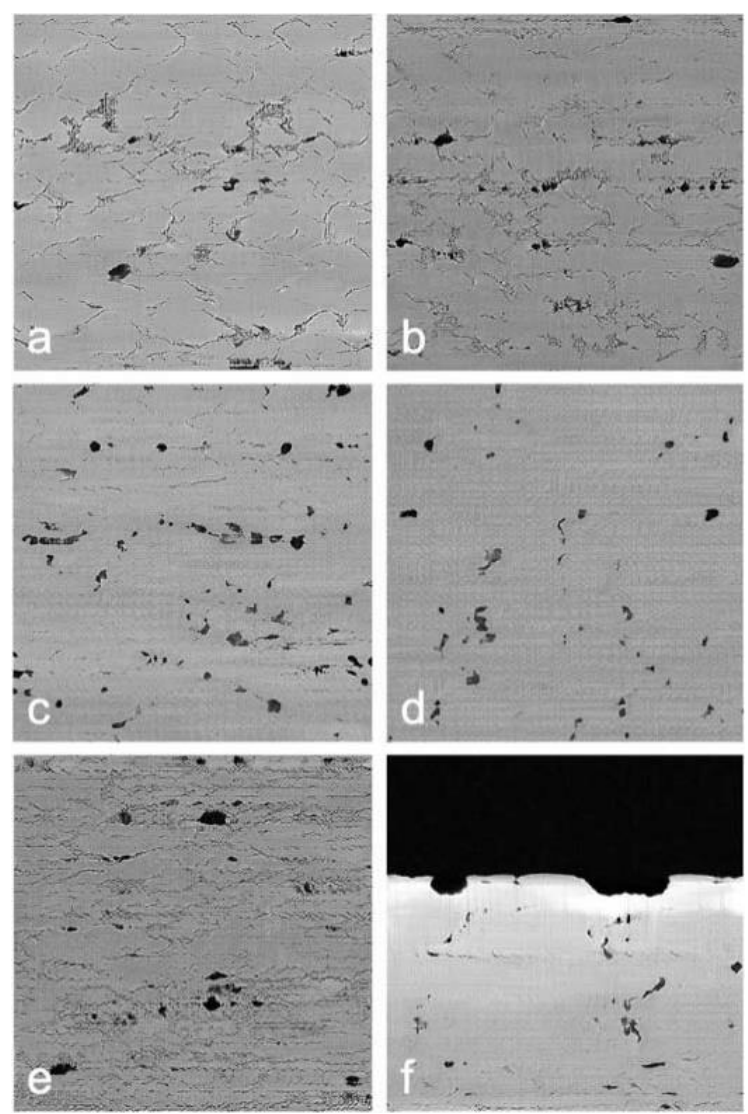

Figure 2. Example synthetic images generated by the trained progressive growing GAN. Images given in (a)-(f) show varying microstructural features, specifically different extent of lamellar transformation products, and distribution of carbides. The micrograph in (f) shows the edge of a fuel plate.

\section{References}

[1] Chowdhury, Aritra, et al. "Image driven machine learning methods for microstructure recognition." Computational Materials Science 123 (2016): 176-187.

[2] Ma, Wufei, et al. "Image-driven discriminative and generative machine learning algorithms for establishing microstructure-processing relationships." Journal of Applied Physics 128.13 (2020): 134901. 